\title{
RETOS A LAS PROTECCIONES ELÉCTRICAS EN LAS REDES DE DISTRIBUCIÓN CON GENERACIÓN DISTRIBUIDA
}

\section{CHALLENGES OF DISTRIBUTED GENERATION ON THE ELECTRIC PROTECTION IN DISTRIBUTION NETWORKS}

\author{
Marta Bravo de las Casas ${ }^{1} \quad$ Yumil Yanez Boza ${ }^{2}$ \\ Recibido: 11 de marzo de 2008, aceptado: 10 de marzo de 2009 \\ Received: March 11, 2008 Accepted: March 10, 2009
}

\begin{abstract}
RESUMEN
Las redes de distribución han sido diseñadas para que la potencia fluya en una sola dirección. La introducción de la Generación Distribuida (GD) hace que esta consideración ya no sea válida. Esto traerá nuevos retos para la operación y el diseño de la red. Una de las áreas críticas más afectadas que existe es la de las protecciones eléctricas.

En este trabajo se hace el estudio de cómo se afectan los ajustes de las protecciones eléctricas al trabajar con generación distribuida en una red de distribución. Para ello se simuló una red con la ayuda del software denominado PSX. Se conectó generación en distintos lugares, de diferentes capacidades y tipos. Se calcularon los niveles de cortocircuitos en los distintos escenarios para los cuales fueron ajustadas las protecciones. Se obtuvieron un conjunto de resultados interesantes, los cuales pueden conducir a nuevas estrategias en lo que respecta a estos equipos.
\end{abstract}

Palabras clave: Corrientes de cortocircuito, generación distribuida, protecciones eléctricas, redes de distribución.

\section{ABSTRACT}

The distribution networks have been designed so that the power flows in a single direction. When the Distributed Generation is introduced this consideration is no longer valid. Distributed Generation will bring new challenges for the operation and the design of the network. One of the most critical areas affected is the electric protection.

This paper discusses issues of how the adjustments of the electric protection are affected when the Distributed Generation is introduced in a distribution network. For this purpose, the network was simulated with the help of the software named PSX. The generation was connected in different places, with different capacities and types. The levels of short circuits were calculated in the different scenarios in which the protections were adjusted. A set of interesting results was obtained, which can lead to new strategies in what concerns to these protections.

Keywords: Short circuit currents, distributed generation, electric protection, distribution networks.

\section{INTRODUCCIÓN}

La conexión de GD en las redes de distribución convierte a sistemas simples en redes complicadas. Los sistemas radiales tendrán ahora múltiples fuentes, las cuales hacen cambiar el flujo de la corriente de falla. Los esquemas de protección tradicionales se convierten en no efectivos [1].

Varios estudios han mostrado los siguientes problemas en las protecciones [2]: operación incorrecta de las protecciones de los alimentadores, incremento o decrecimiento de los niveles de cortocircuito [3-4], pérdida de sensibilidad, aislamiento no deseado, recierre no sincronizado, etc.

Los esquemas de protección que existen en estas redes son generalmente de sobrecorriente tiempo inverso o tiempo definido, de acuerdo a los criterios convencionales de la protección [5-8].

El sistema de protección tradicional ha sido diseñado considerando los niveles de los cortocircuitos, corrientes de máxima carga, sistema de voltaje y nivel de aislamiento

\footnotetext{
1 Centro de estudios Electroenergéticos. Facultad de Ingeniería Eléctrica. Universidad Central "Marta Abreu de Las Villas. Carretera de Camajuaní km 51/2 Santa Clara. Villa Clara. Cuba. E-mail: mbravo@uclv.edu.cu; mbravocasas@yahoo.com

2 Empresa Constructora para la Industria Eléctrica (ECIE) de Villa Clara. Circunvalación e/ Carretera de Sagua y Línea. Santa Clara. Villa Clara. Cuba.
} 
de un sistema radial [9]. Después de la conexión de la GD, parte del sistema puede que no sea radial, lo que trae consigo problemas en la actuación de las protecciones. Pueden ocurrir operaciones incorrectas, fallo del esquema de los fusibles, reducción del alcance potencial de cortocircuitos a localizar por la protección así como disparos incorrectos, además de problemas con la coordinación.

El efecto de la GD en la coordinación depende del tamaño, tipo y conexión de la fuente que se conecte. Puede ocurrir que aparezcan altos tiempos de limpieza de falla o que exista coordinación no selectiva, ambas no aceptadas por los criterios de protección y además por el mercado de la energía eléctrica [8].

La protección opera innecesariamente para fallas en otras zonas de la protección, producto de la contribución de la GD. En la figura 1 se muestra un ejemplo. El relé ubicado en A y el restaurador (recerradores o recloser) no son direccionales. Para el caso de falla en B puede ocurrir un disparo incorrecto de A por la contribución de la GD, por lo tanto se nota la necesidad de que las protecciones sean direccionales.

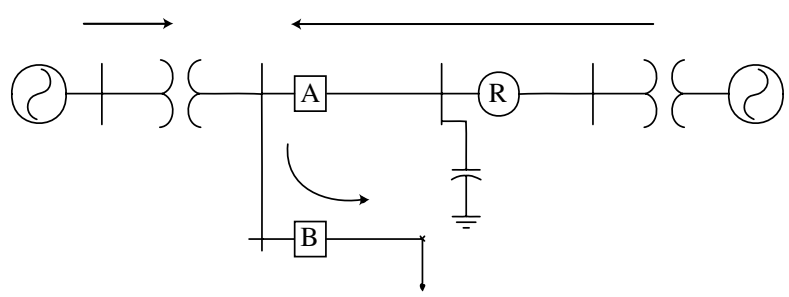

Figura 1. Esquema ilustrativo de mala operación cuando hay GD.

Muchas empresas utilizan esquemas de protección con restauradores [5-7] y fusibles. Normalmente el primero y/o segundo disparo del restaurador es rápido, y los otros dos con retardo para lograr una buena coordinación con los fusibles. En los dos primeros disparos el fusible no debe fundirse, para si el cortocircuito es transitorio. La presencia de DG puede que haga que esto no se cumpla debido al sentido de circulación de la corriente.

Además, la utilización de GD puede provocar que el funcionamiento del restaurador no sea exitoso, ya que la GD continúa en operación durante el tiempo en que el restaurador está abierto. El voltaje se mantiene y la falla se seguirá alimentado, lo que puede hacer que el arco no se extinga y por lo tanto la falla se convierte en permanente. Esto se traduce en un deterioro de la fiabilidad de la red y un incremento del número de consumidores que han perdido el servicio. Por otro lado, el recierre no exitoso incrementa el daño a las componentes de la red ya que el restaurador cerrará en el momento en que la falla está presente [1].
Otro problema es la falta de sensibilidad de la protección de sobrecorriente para fallas dentro de la zona de operación, causado por la reducción del alcance del dispositivo de protección del alimentador. La sensibilidad de la protección se reduce cuando existe GD entre el dispositivo y la falla. Esto se debe a que la GD sube el perfil del voltaje en la parte del alimentador en cuestión, lo cual reduce la corriente vista por el relé y reduce la sensibilidad, de manera que la falla tiene que estar más cercana a la protección para que la detecte.

En [10] se expone el efecto de la GD en la coordinación de los equipos de protecciones tal como: fusibles-fusibles, fusibles-restauradores y relé-relé. En cada caso, en dependencia del tamaño y colocación de la GD, hay márgenes en el que la coordinación sería posible pero en otros casos no.

En [11] se expone un caso de estudio de la aplicación de un relé electromecánico o electrónico en un circuito de distribución radial contra la aplicación de un relé multifuncional digital. Se brindan las ventajas del tener una protección multifuncional numérica en los lugares donde se adiciona DG en las redes de distribución radiales.

\section{ANÁLISIS TEÓRICO}

\section{Estado de las protecciones en las redes de distribución}

En Cuba las redes de distribución se encuentran en un proceso de descapitalización debido al embargo económico al que ha estado sometido el país por el gobierno de los Estados Unidos, por más de 40 años y que ha sido más fuerte a partir de 1990.

Lo anterior ha traído como consecuencias que se confronten un conjunto de problemas técnicos, pues no se han podido modernizar las redes como resultaría necesario, de acuerdo al desarrollo tecnológico de nuestros días. En particular, las protecciones de las redes de distribución han sufrido este deterioro.

A principios de la década de los 90 del pasado siglo estos circuitos se encontraban protegidos con relés de diferentes marcas entre ellas rusas e incluso algunos IAC norteamericanos (General Electric) que aún se comercializan, del tipo electromagnéticos. Existían en algunos casos restauradores de diferentes firmas. Pero con el tiempo estas protecciones se vieron muy afectadas tanto en sus posibilidades de brindar protección como 
que se dañaron producto de roturas y el deterioro normal del tiempo.

Se ha introducido, por lo tanto, un conjunto de mejoras técnicas en las redes de distribución que alcanzan a las protecciones eléctricas. Cada uno de los circuitos se encuentran protegidos de formas diferentes actualmente, por ejemplo, con relés microprocesados de la Basler [12] de primera generación [13-14], otros por relés digitales modernos de grandes posibilidades de protección, los denominados Nu-Let [15].

Se tiene como lineamiento nacional por problemas con las protecciones, solo una al inicio del circuito y se han eliminado todos los fusibles a lo largo del circuito, solo se protegen con fusibles los primarios de los transformadores, y no se aplican en puntos importantes de la red, como expone toda la bibliografía específica sobre el tema de distribución [5-7].

Es de vital importancia tener un relé digital a la salida de la subestación. Entre las ventajas de la protección de sobrecorriente numérica multifuncional en la subestación está que la misma tiene varias formas de curvas con lo que se logra una mejor coordinación, más un amplio intervalo de valores de corrientes de arranque los cuales no son posibles en los otros relés. Además de la posibilidad de seleccionar grupos de ajustes dependiendo de la condición de operación [11].

En caso de tener GD en la red será necesario un número mayor de funciones de protección, para lo cual con las versiones electromagnéticas habría que introducir varios relés. Con la versión digital multifuncional se logra con un solo equipo, donde el costo es aproximadamente la mitad de los que llevaría en caso de hacer las funciones por separado. A lo que se suma las posibilidades de estos equipos modernos de registros de eventos, oscilogramas, etc. [13-14]

\section{Características del circuito analizado}

Circuito \# 19 de la Ciudad de Santa Clara, 13,8 kV. Subestación Roble. Carga predominante: residencial. Número de cargas: 109, donde las más importantes son el Hospital Oncológico Celestino Hernández y el Cardiocentro. Recorre una zona bastante extensa, 3,02 $\mathrm{km}$ y un área de $4,56 \mathrm{~km}^{2}$. Está alimentado por una línea de $34,5 \mathrm{kV}$ desde la subestación Santa Clara Industrial $(110 / 34,5 \mathrm{kV})$ donde se encuentran instalados dos grupos de baterías de 8 Generadores Diesel de 2,36 MVA. Se encuentra protegido por una protección numérica moderna, el Nu-Lec.
En el circuito analizado se le implementaron tres opciones en cuanto a la generación a la entrada de los mismos.

$1^{\text {ra }}$ Opción: como se encontraba el sistema antes que se implementara la generación distribuida.

$2^{\text {da }}$ Opción: están generando el sistema y la GD instalada en Santa Clara Industrial, situación actual.

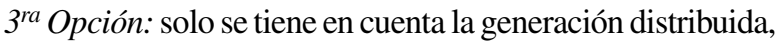
es decir, este puede ser el caso cuando el sistema se encuentra afectado por algún factor atmosférico, ciclones, huracanes, etc. Es decir, con la GD de Santa Clara Industrial.

Adicionalmente se consideraron variantes en las opciones: que pudiera existir una batería en la subestación de distribución de 8 generadores de 2,36 MVA cada uno y que los grupos de emergencia del Hospital Celestino Hernández de 220 KVA y del Cardiocentro de 440 KVA puedan sincronizarse al sistema.

\section{METODOLOGÍA UTILIZADA EN EL AJUSTE DE LAS PROTECCIONES}

\section{Ajuste del arranque del tiempo inverso de fase [5]-[7]}

La corriente de arranque del relé que se ubica en la fase se calcula de acuerdo a la expresión:

$$
\text { Iar }=K \cdot \frac{\text { Icmáx }}{\mathrm{N}_{\mathrm{TC}}}
$$

Donde: $\mathrm{K}=1,5$ e Ic ${ }_{\text {máx }}$ es la corriente máxima de carga que puede circular por la protección y $\mathrm{N}_{\mathrm{TC}}$ es la relación de transformación del transformador de corriente.

\section{Chequeo de Sensibilidad}

Se debe hacer con la corriente de cortocircuito mínima, Icc $_{\text {mín }}$ que puede circular por el transformador de corriente (TC) en el punto más alejado eléctricamente.

$$
K s=\frac{I c c \min }{I a r \cdot N t c}
$$

El valor de Ks de la expresión (2) debe ser mayor que 1,5 para que el relé brinde sensibilidad a toda la línea. 


\section{Ajuste de arranque del instantáneo de fase [5-7]}

La corriente de arranque del relé que se ubica en tierra se calcula de acuerdo a la expresión:

$$
\text { Iar }=K \cdot \frac{\text { Iccmáx }}{N t c}
$$

Donde: $\mathrm{K}=1,3$ y de los datos obtenidos del PSX se tiene la corriente máxima, Icc $_{\text {máx }}$, que es la mayor corriente de cortocircuito que por el TC puede circular en el punto más cercano a la protección.

\section{Ajuste del arranque del tiempo inverso de tierra [5-7]}

Como el tiempo inverso de tierra es más rápido que el de fase se emplea la siguiente expresión:

$$
\text { Iar }=0,1 \div 0,2 \operatorname{In}_{\mathrm{TC}}
$$

donde: $\mathrm{In}_{\mathrm{TC}}$ es la corriente nominal por el transformador de corriente que es $5 \mathrm{~A}$.

En el chequeo de sensibilidad y el ajuste del instantáneo de tierra se utilizarán las expresiones (2) y (3) teniendo en cuenta que los cortocircuitos mínimos y máximos son a tierra.

\section{Selección de las curvas Tiempo Corriente (TC)}

Se seleccionaron las curvas de trabajo de los dispositivos para todos los circuitos en todas sus opciones y variantes de manera que cumplieran con los requerimientos técnicos de las protecciones eléctricas [5-7]. Siempre la protección primaria debe actuar antes que el respaldo.

Para la selección del tipo de curva, se tomó un criterio dado por la Unión Eléctrica de Cuba de que debe cumplirse que el múltiplo de la corriente de arranque del relé para $1 \mathrm{~s}$ debe ser:

$$
M=I_{c c \text { máx }} / 4 I_{a p}
$$

Donde $\mathrm{Icc}_{\text {máx }}$ es la corriente máxima de cortocircuito que circula por el relé para una falla en el punto más cercano eléctricamente, e $\mathrm{I}_{\text {ap }}$ es la corriente de operación por primario.

\section{RESULTADOS Y DISCUSIÓN}

Como dato particular de este circuito, se tiene que: $\mathrm{N}_{\mathrm{TC}}=200 / 5=40, \mathrm{Sc}_{\text {máx. }}=5793 \mathrm{kV} . \mathrm{A}$ (demanda del circuito). Todos los cálculos de cortocircuitos se hicieron utilizando el software PSX confeccionado por el Centro de Estudios Electroenergéticos de la Universidad Central "Marta Abreu de Las Villas (UCLV) [16]. Los resultados de los ajustes de las protecciones para cada opción y variante se muestran en las tablas 1, 2 y 3 .

Tabla 1. Ajustes de las protecciones. $1^{\text {ra }}$ opción (Sistema).

\begin{tabular}{|l|c|c|c|c|c|c|}
\hline \multirow{2}{*}{ Variante } & \multicolumn{3}{|c|}{ Fase } & \multicolumn{3}{c|}{ Tierra } \\
\cline { 2 - 7 } & Tiempo inverso & Instantáneo & Tiempo inverso & Instantáne0 \\
\cline { 2 - 7 } & $\mathbf{I a r ( A )}$ & Ks & $\operatorname{Iar(A)}$ & $\operatorname{Iar(A)}$ & Ks & Iar(A) \\
\hline Sin GD & 9,1 & 2,245 & 37,64 & 1 & 24 & 15,145 \\
\hline $\begin{array}{l}\text { Con las baterías } \\
\text { conectadas } \\
\text { y el grupo } \\
\text { Cardiocentro }\end{array}$ & 9,1 & 5,06 & 156,94 & 1 & 30,1 & 96,7 \\
\hline $\begin{array}{l}\text { Con las baterías } \\
\text { conectadas y el } \\
\text { grupo hospital }\end{array}$ & 9,1 & 5,114 & 156,94 & 1 & 37,4 & 100,6 \\
\hline $\begin{array}{l}\text { Con toda la GD } \\
\text { conectada }\end{array}$ & 9,1 & 4,83 & 156,94 & 1 & 35,3 & 101,7 \\
\hline
\end{tabular}

Tabla 2. Ajustes de las protecciones. $2^{\text {da }}$ opción (Sistema+GD).

\begin{tabular}{|l|c|c|c|c|c|c|}
\hline \multirow{3}{*}{ Variante } & \multicolumn{3}{|c|}{ Fase } & \multicolumn{3}{c|}{ Tierra } \\
\cline { 2 - 7 } & Tiempo inverso & Instantáneo & Tiempo inverso & Instantáneo \\
\cline { 2 - 7 } & Iar(A) & Ks & $\operatorname{Iar(A)}$ & Iar(A) & Ks & Iar(A) \\
\hline Sin GD & 9,089 & 2,27 & 38,22 & 1 & 321 & 601 \\
\hline $\begin{array}{l}\text { Con las baterías } \\
\text { conectadas } \\
\text { y el grupo } \\
\text { Cardiocentro }\end{array}$ & 9,089 & 5,07 & 157,6 & 1 & 401 & 2982 \\
\hline $\begin{array}{l}\text { Con las baterías } \\
\text { conectadas y el } \\
\text { grupo hospital }\end{array}$ & 9,089 & 5,12 & 157,6 & 1 & 498 & 3101 \\
\hline $\begin{array}{l}\text { Con toda la GD } \\
\text { conectada }\end{array}$ & 9,089 & 4,83 & 157,6 & 1 & 407 & 3134 \\
\hline
\end{tabular}

Tabla 3. Ajustes de las protecciones. $3^{\text {ra }}$ opción (Solo GD).

\begin{tabular}{|l|c|c|c|c|c|c|}
\hline \multirow{2}{*}{ Variante } & \multicolumn{3}{|c|}{ Fase } & \multicolumn{3}{c|}{ Tierra } \\
\cline { 2 - 7 } & Tiempo inverso & Instantáneo & \multicolumn{1}{c|}{ Tiempo inverso } & Instantáne0 \\
\cline { 2 - 7 } & Iar(A) & Ks & $\operatorname{Iar(A)}$ & Iar(A) & Ks & Iar(A) \\
\hline Sin GD & 9,089 & 1,26 & 18,95 & 1 & 12,3 & 3,695 \\
\hline $\begin{array}{l}\text { Con las baterías } \\
\text { conectadas } \\
\text { y el grupo } \\
\text { Cardiocentro }\end{array}$ & 9,089 & 5,04 & 155,32 & 1 & 30 & 96 \\
\hline $\begin{array}{l}\text { Con las baterías } \\
\text { conectadas y el } \\
\text { grupo hospital }\end{array}$ & 9,089 & 5,1 & 155,32 & 1 & 37,28 & 99,87 \\
\hline $\begin{array}{l}\text { Con toda la GD } \\
\text { conectada }\end{array}$ & 9,089 & 4,8 & 155,32 & 1 & 30,45 & 101,01 \\
\hline
\end{tabular}


Al correr esta tercera opción, en su primera variante ( $\sin$ GD en la subestación), se puede observar de la tabla 3 que el relé no es sensible $(K s=1.26), K s$ está por debajo del valor mínimo 1,5 , que sería el por ciento de seguridad que se tiene para incluir el arco en el cortocircuito.

Es necesario conocer hasta dónde el relé es capaz de brindar sensibilidad a las líneas que sería el punto crítico para lograr la condición de sensibilidad, según:

$$
\text { Icc } \min \geq K s \cdot I a r \cdot N t c
$$

Sustituyendo: Icc $\min \geq 545.88 \mathrm{~A}$

Utilizando el software PSX se obtuvo que en el nodo 2, el más próximo al relé, el nivel de cortocircuito mínimo es 505 A, el cual está por debajo del valor crítico calculado.

Para resolver este problema se pueden implementar varias soluciones posibles.

Una primera variante posible es instalar un segundo relé (relé 2), en el nodo 3 del mismo tipo que el anterior, como se muestra en la figura 2, con lo que es posible mejorar los factores a tener en cuanta a la hora de seleccionar una protección, en particular la sensibilidad, que es el factor más crítico del relé 1 .

No se propone poner un fusible en este punto pues la condición de carga máxima es mayor a 200 A, y el fusible mayor que se dispone es el $200 \mathrm{~K}$.
Los parámetros de ajuste de la protección quedaron como se muestran en la tabla 4 .

Tabla 4. Ajustes de la protección 2. Primera variante.

\begin{tabular}{|l|c|c|c|c|c|c|}
\hline \multirow{4}{*}{ Variante } & \multicolumn{3}{|c|}{ Fase } & \multicolumn{3}{c|}{ Tierra } \\
\cline { 2 - 7 } & Tiempo inverso & Instantáneo & Tiempo inverso & Instantáneo \\
\cline { 2 - 7 } & Iar(A) & Ks & $\operatorname{Iar(A)}$ & $\operatorname{Iar(A)}$ & Ks & Iar(A) \\
\hline $1^{\text {a }}$ Sin GD & 8,5 & 1,49 & 18,66 & 1 & 14,1 & 6,44 \\
\hline
\end{tabular}

El factor de sensibilidad no es el establecido, pero se pudiera implementar, pues está muy cercano al valor establecido. Por lo tanto, se pasa a verificar hasta dónde es capaz de proteger el nuevo dispositivo de protección, encontrándose que es el nodo 10, que no es el final del circuito. Se tendrá que conectar en ese nodo otro dispositivo de protección, por ejemplo un fusible para ver si este es capaz de proteger el resto del circuito. El fusible necesario en este caso resultó ser un $80 \mathrm{~K}$ que cumple con todos los requerimientos de protección para este dispositivo [5-7]. Esta variante resultaría factible, sólo que los dos relés se encuentran ligeramente por debajo de la sensibilidad ideal.

Una segunda variante es colocar el relé 2 un poco más alejado de la subestación, en el nodo 10 , figura 3 , esto trae como inconveniente que el relé 1 no cumple con el requisito de sensibilidad $(K s=1,34), 3 \%$ aproximadamente por debajo. Pero el relé 2 lograría proteger a todo el circuito como se pudo comprobar y se ahorra un dispositivo de protección.

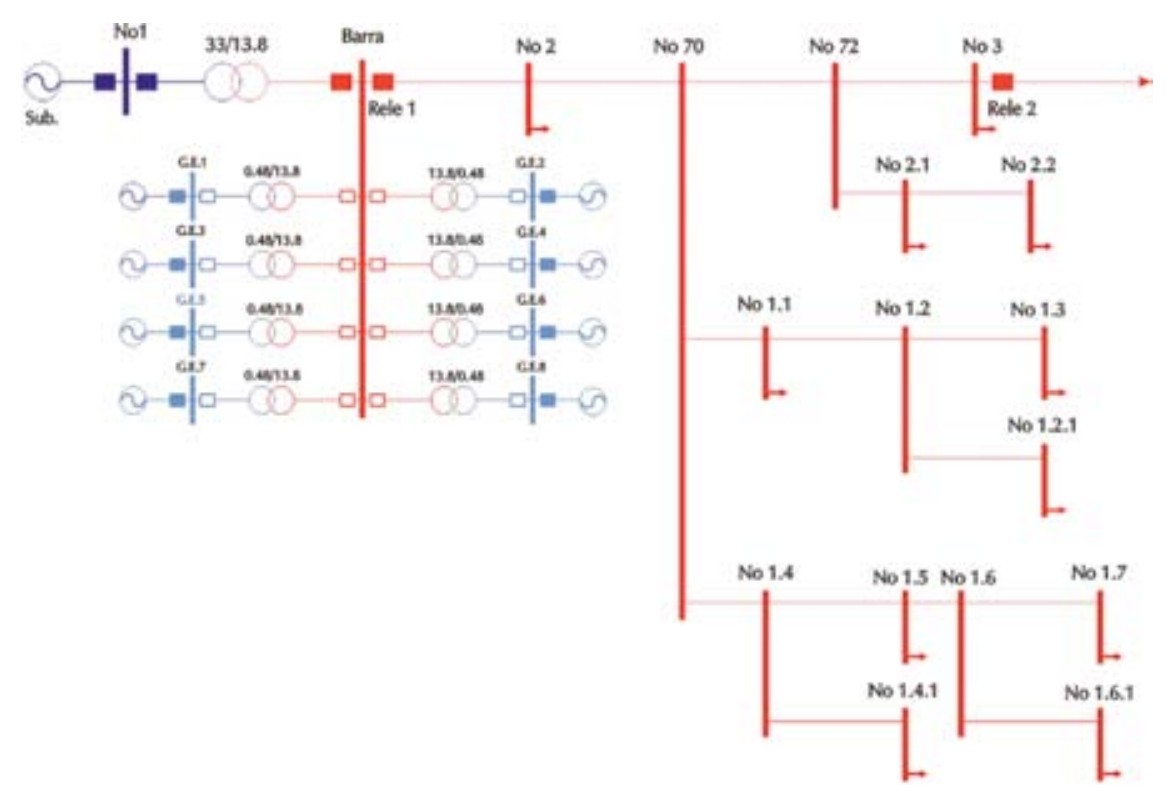

Figura 2. Posible solución para la falta de sensibilidad del relé. Primera variante. 


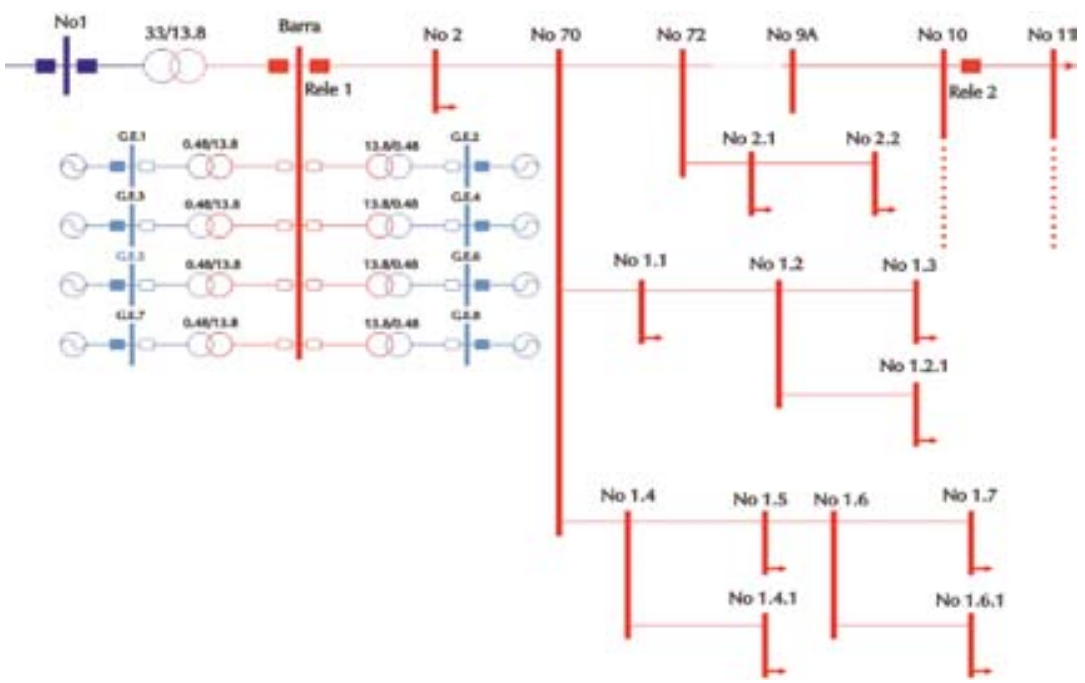

Figura 3. Monolineal para la segunda variante propuesta con el objetivo de lograr sensibilidad

Los parámetros de ajuste de este dispositivo nuevo se muestran en la tabla 5 .

Tabla 5. Ajustes de la protección 2. Segunda variante.

\begin{tabular}{|l|c|c|c|c|c|c|}
\hline \multirow{3}{*}{ Variante } & \multicolumn{3}{|c|}{ Fase } & \multicolumn{3}{c|}{ Tierra } \\
\cline { 2 - 7 } & Tiempo inverso & Instantáne0 & Tiempo inverso & Instantáneo \\
\cline { 2 - 7 } & $\operatorname{Iar}(\mathbf{A})$ & Ks & $\operatorname{Iar(A)}$ & $\operatorname{Iar(A)}$ & Ks & $\operatorname{Iar(A)}$ \\
\hline $1^{\text {a }}$ Sin GD & 6,81 & 1,69 & 18,27 & 1 & 13,2 & 6,05 \\
\hline
\end{tabular}

Como se puede observar de la tabla 5 el relé ahora es sensible hasta el final del circuito.

\section{Selección de la característica TC y coordinación en los casos necesarios [5-7]}

Del catálogo del fabricante de la protección Nu-Lec [15] se tienen las ecuaciones que describen las características moderadamente, muy y extremadamente inversa.

Con el criterio mencionado, expresado por la expresión (5) se obtuvieron las características TC de cada una de las opciones y variantes de los relés de fase y tierra. Se escogió una característica tiempo muy inverso.

Solo para la $3^{\text {era }}$ opción, donde no existe sensibilidad, fue necesario realizar la coordinación dado por la necesidad de ubicar dispositivos de protección a lo largo del circuito.

En el caso de la primera variante es necesario hacer primero la coordinación entre el relé 2 y el fusible y luego entre los dos relés, tal como plantea la literatura. El intervalo selectivo se escogió de 0,4 s. El resultado se muestra en las figuras 4 y 5 .

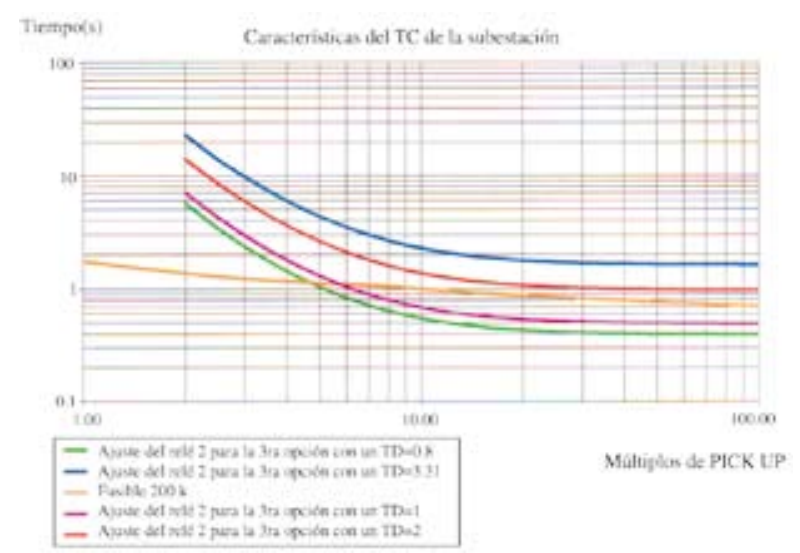

Figura 4. Coordinación relé 2 y Fusible, variante 1.



Figura 5. Coordinación relé 1 y relé 2 , variante 1 .

En el caso de la segunda variante solo fue necesario hacer la coordinación entre relés. 


\section{CONCLUSIONES}

Se pudo demostrar que cuando la GD se conecta en la subestación de subtransmisión junto con el sistema, trae consigo un aumento considerable de los niveles de cortocircuitos, haciendo más sensibles las protecciones de sobrecorriente, tanto de fase como de tierra, pero trae variaciones de los ajustes de acuerdo al escenario con el cual se vaya a operar la red.

Resulta imprescindible tener protegidos los circuitos de distribución con relés digitales, debido a que las corrientes de cortocircuito en la localización del relé pueden aumentar producto de la entrada del grupo de emergencia, o baterías que entren en los picos a generar en conjunto al sistema, o puede también disminuir producto a la generación intermedia o por la generación sin el sistema electroenergético, es decir, en isla. Además de posibles cambios de dirección de estas corrientes de cortocircuito de acuerdo a donde esté la GD y su capacidad. Es por ello que se necesitan más posibilidades en los juegos de ajustes y de curvas para lograr la coordinación necesaria con los demás dispositivos de protección.

\section{REFERENCIAS}

[1] L. Kumpulainen and K. Kauhaniemi. "Distributed generation and reclosing coordination". Nordic Distribution and Asset Management Conference 2004. Fecha de consulta: Abril 2006. URLs: http:// powersystems.tkk.fi/nordac2004/papers/ nordac2004_kumpulainen_et_kauhaniemi_paper. pdf

[2] G. Koeppel. "Distributed generation. Literature review and outline of the Swiss situation". EEH Power Systems Laboratory. ETH Swiss Federal Institute of Technology Zurich. Internal Report, p. 19. November 2003.

[3] N. Nimpitiwan. "Fault current issues for market driven power systems with distributed generation". IEEE PES General Meeting, San Francisco, p. 7. June 2005.

[4] H. Altuve. "Protección de redes eléctricas". Ministro de Educación Superior. Universidad Central de Las Villas, p. 254. Cuba. 1990.

[5] ABB Power T\&D Company Inc. Relay Division Coral Spring, Florida. "Protective relaying. Theory and applications". Edited by W. A. Elmore, M. Dekker, INC, New York, Basel, Hong Kong, p. 267. 1994.
[6] J.L. Blackburn. "Protective relaying. Principles and applications". M. Dekker, INC, New York, p. 545.1987.

[7] J. Jäger, T. Keil, L. Shang and R. Krebs. "New protection coordination methods in the presence of Distributed Generation". 8th International Conference on Developments in Power System Protection, Amsterdam (The Netherlands), p. 4. April 2004.

[8] Y. Baghzouz. "Voltage regulation and overcurrent protection issues in distribution feeders with Distributed Generation. A Case Study". Proceedings of the 38th Hawaii International Conference on System Sciences, Hawaii, p. 7. 2005.

[9] Girgis and S. Brahma. "Effect of distributed generation on protective device coordination in distribution system". Clemson University Electric Power Research Association, ECE Department, USA, p. 119. 2001.

[10] G. Dalke. Basler Electric Company. "Protective relaying when Distributed Generation is added to your circuit". 2002, p. 14. Fecha de consulta: Abril 2006. URLs: http://basler.com/ downloads/ ProtwDG.pdf

[11] Basler Electric. Catálogo del fabricante de las Protecciones. "Instruction manual for overcurrent". Type BE1-50/51M, Highland, Illinois, p. 30. 03/1995.

[12] A.G. Phadke and J.S. Thorp. "Computer Relaying for Power Systems". John Wiley \& Sons Inc. Research Studies Press LTD, p. 289. England. 1988.

[13] P. Montané. "Protecciones en las Instalaciones. Evolución y Perspectivas". Segunda edición. Marcombo. Boixareo Editores, p. 8. Barcelona, España. 1998.

[14] Nu-Lec Industries. A company Schneider Electric. Manual Técnico Restaurador. N15/N27/N38, Litton, Australia, p. 170. October 2006.

[15] Z. García y J. González Cueto. "Manual de usuario del PSX". Formato Electrónico. Intranet de la Facultad de Ingeniería Eléctrica, p. 70. 2002. URLs: http://intranet.fie.uclv.edu.cu 